Introduction/Background Malignant sex cord stroma cell tumours (SCST) account for less than eight percent of ovarian malignancies. The Arbeitsgemeinschaft fuer Gynaekologische Onkologie (AGO) has established a clinicopathological (Current Ovarian geRm cell and SEx cord stromal Tumour Treatment strategies, CORSETT) database for a better documentation and understanding of this rare disease. Here, we present the first clinicopathological descriptive analysis for patients with independently confirmed SCST from the CORSETT database.

Methodology 20 German centres entered mixed retro- and prospective data of SCST patients with tumour specimens available treated between 2000 to 2014 into the CORSETT database. An independent CORSETT pathology reference panel checked the primary histological diagnosis.

We conducted a descriptive analysis of the treatment strategies and created Kaplan-Meier curves and cox regression analyses for the survival analysis.

Results The reference pathology panel diagnosed 143 patients with granulosa cell (GCT, FIGO stage $\mathrm{I}=120,87.0 \%$ ) and 14 patients with Sertoli-Leydig cell (SLCT, FIGO stage I = $11,91.7 \%$ ) tumours (others $=5$ ). The median age of patients with GCT was 57.6 years (SLCT: 47.2 years). 87 of GCT $(61.7 \%)$ and eight SLCT $(57.1 \%)$ patients were treated with laparoscopy and the tumour ruptured intraoperatively in $22 \%$ (SLCT: $7.7 \%)$ of the cases. 57 GCT (45\%) and eight SLCT $(57 \%)$ patients received fertility-sparing surgery. 19 of GCT $(15 \%)$ and two SLCT $(15.4 \%)$ patients received adjuvant chemotherapy. 59 of GCT (45\%) and two of SLCT (14.3\%) patients experienced a disease recurrence. The median progression-free survival (PFS) for all SCST patients was 80.4 months, (overall survival not reached). Advanced FIGO stage was associated with decreased PFS ( $p<0.05$ ).

Adjuvant chemotherapy had no statistically significant beneficial effect on PFS (all regimens $\mathrm{p}>0.05$ ).

Conclusion In this analysis, almost every fourth SCST patient treated surgically experienced an intraoperative cyst rupture that had however no impact on disease recurrence. One in five SCST patient received adjuvant chemotherapy that had no PFS improvement.

Disclosures

\section{TARGETING AKT AND DNA-PK AS A THERAPEUTIC STRATEGY IN PLATINUM RESISTANT HIGH-GRADE SEROUS OVARIAN CANCER}

${ }^{1}$ Natasha Rinne, 'Shengnan Yuan, ${ }^{1}$ Christina Fotopoulou, ${ }^{2}$ Hani Gabra, ${ }^{1}$ Paula Cunnea. ${ }^{1}$ Imperial College London; Division of Cancer, Department of Surgery and Cancer; ${ }^{2}$ Bergenbio, Magdalen Centre; Robert Robinson Avenue, The Oxford Science Park

\subsection{6/ijgc-2020-ESG0.112}

Introduction/Background High-grade serous ovarian cancer (HGSOC) is the most lethal form of gynaecological malignancy. Despite initial sensitivity to platinum chemotherapy, the majority of patients develop resistance to treatment and eventually die. Current treatment options for platinum-resistant patients are limited.

The role of the PI3K/AKT/mTOR pathway has been described in chemo-resistant HGSOC, in particular through activation of AKT by DNA-PK in response to platinum treatment. As increasing numbers of AKT and DNA-PK inhibitors advance to clinical trials, determining mechanism of action and efficacy is crucial.
This project aims to evaluate inhibition of AKT or DNA$\mathrm{PK}$ as a therapeutic strategy to target platinum resistance in HGSOC, and identify proteomic signatures confirming mechanism of action and target inhibition.

Methodology A panel of seven AKT and DNA-PK inhibitors were tested in combination with cisplatin chemotherapy in immortalised HGSOC cell lines and primary tumour cells cultured from HGSOC tumour/ascites samples. Clonogenic assays were performed to establish effect of inhibitor treatment in combination with cisplatin chemotherapy on the ability of cells to form colonies. Isobologram assays were performed to establish synergy/antagonism between inhibitors and cisplatin chemotherapy. Proteomic Reverse Phase Protein Array (RPPA) was performed to determine the mechanism of action of inhibitors, and results were confirmed with immunoblotting.

Results Treatment with AKT or DNA-PK inhibitors in combination with cisplatin led to significantly enhanced apoptotic responses in immortalised platinum-resistant HGSOC cell lines $(n=5)$, and in primary cells derived from ascites or tumour $(\mathrm{n}=4, \mathrm{p}<0.01, \mathrm{p}<0.05)$, compared to cisplatin treatment alone. In platinum-resistant HGSOC cell lines, fewer cell colonies were observed with increasing concentrations of AKT or DNA-PK inhibitors in combination with cisplatin $(n=3)$ in comparison with cisplatin alone. Varying synergistic effects were observed across the panel of inhibitors when combined with cisplatin; Uprosertib (AKT inhibitor) in particular displayed strong synergy with cisplatin (Loewe analysis). Proteomic analysis of inhibitor treatment in HGSOC platinumresistant cells demonstrated the mechanism of action of Uprosertib in targeting the PI3K/AKT pathway.

Conclusion In platinum-resistant HGSOC cells, AKT or DNAPK inhibition functioned synergistically with cisplatin and reduced cell growth and proliferation. In both immortalised and primary HGSOC cell lines tested, AKT or DNA-PK inhibition significantly enhanced the apoptotic response to cisplatin demonstrating the efficacy of AKT or DNA-PK as potential therapeutic targets in chemoresistant HGSOC. By improving patient response to treatment, AKT and DNA-PK inhibitors could expand the therapeutic options for patients with platinum-resistant HGSOC, improving overall survival.

Disclosures CF: advisory boards and honoraria from Roche, Tesaro, Sequana, Olympus, Astra Zeneca. Other authors have no conflict of interest.

\section{TREATMENT STRATEGIES AND SURVIVAL OF WOMEN WITH MALIGNANT OVARIAN GERM CELL TUMOURS - AN ANALYSIS OF THE AGO-CORSETT DATABASE}

${ }^{1}$ Annette Hasenburg, ${ }^{2}$ Helmuth Plett, ${ }^{3}$ Philipp Harter, ${ }^{4}$ Stefan Kommoss, ${ }^{5}$ Jaqueline Keul, ${ }^{6}$ Eva Roser, ${ }^{7}$ Bastian Czogalla, ${ }^{8}$ Michaela Bossart, ${ }^{9}$ Theresa Link, ${ }^{10}$ Maximilian Klar. ${ }^{1}$ Dept Gynecology and Obstetrics; University Center Mainz; Klinik und Poliklinik für Geburtshilfe und Frauengesundheit; ${ }^{2}$ Klinikum Essen Mitte; ${ }^{3}$ Klinikum Essen Mitte; Gynaecology and Gynaecological Oncology; ${ }^{4}$ Universitätsklinikum Tübingen; Universitätsfrauenklinik; University Hospital Tuebingen; ${ }^{5}$ University Hospital Tuebingen; Universitätsfrauenklinik; ${ }^{6}$ Charite Berlin; ${ }^{7}$ MUU Munich; Obstetrics and Gynaecology; ${ }^{8}$ Universitätsklinikum Freiburg; University of Freiburg; Obstetrics and Gynaecology; ${ }^{9}$ Uniklinik Dresden; Gynäkologie und Geburtshilfe; Obstetrics and Gynaecology; ${ }^{10}$ University of Freiburg

\subsection{6/ijgc-2020-ESGO.113}

Introduction/Background Malignant ovarian germ cell tumours (OGCT) account for about five percent of all ovarian malignancies in Western countries. The Arbeitsgemeinschaft fuer 
Gynaekologische Onkologie (AGO) has established a clinicopathological (Current Ovarian geRm cell and SEx cord stromal Tumour Treatment strategies, CORSETT) database for a better documentation and understanding of this rare disease. Here, we present the first descriptive analysis for patients with confirmed OGCT from the CORSETT database.

Methodology 20 German centres entered mixed retro- and prospective data of OGCT patients with histology specimens available treated between 2000 to 2014 into the CORSETT database. An independent CORSETT pathology reference panel checked the primary histological diagnosis. We conducted a descriptive analysis of the treatment strategies and created Kaplan-Meier curves and cox regression analyses for the survival analysis.

Results The reference pathology panel diagnosed 36 patients with dysgerminoma (FIGO stage $\mathrm{I}=77.8 \%$ ), 20 patients with mixed OGCT (FIGO stage $\mathrm{I}=66.7 \%$ ) and 21 with malignant teratoma (FIGO stage $\mathrm{I}=85 \%$ ). The median age of patients with dysgerminoma was 30.2 years (mixed OGCT: 35.6 and teratoma 36.4 years). 23 of dysgerminoma (63.8\%), six of mixed OGCT (31.6\%) and eight of teratoma (38.1\%) patients were treated with laparoscopy and the tumour ruptured intraoperatively in 21\% (dysgerminoma, mixed OGCT: 50\%, teratoma: $22 \%$ ) of the cases. 29 dysgerminoma (85.6\%), 15 mixed OGCT (78.9\%) and 17 teratoma (85\%) patients received fertility-sparing surgery. 20 of dysgerminoma (57\%), 14 of mixed OGCT (70\%) and 11 of teratoma (55\%) patients received adjuvant chemotherapy which decreased the likelihood of disease recurrence significantly in mixed OGCT patients to the highest degree (hazard ratio $=0.21,95 \%$ confidence interval $0.04-0.97)$. In total, two dysgerminoma (4.6\%), nine mixed OGCT (45\%) and three teratoma (14.3\%) patients experienced disease recurrence. The median progression-free and overall survival was not reached in the total cohort of OGCT patients.

Conclusion In this analysis, OGCT patients had an excellent prognosis despite non-negligible rates of intraoperative tumour spillages. Adjuvant chemotherapy appeared to prevent disease recurrence.

Disclosures

\section{PATIENT-REPORTED OUTCOMES (PROS) IN PATIENTS (PTS) RECEIVING NIRAPARIB IN THE PRIMA/ENGOT- OV26/GOG-3012 TRIAL}

\begin{abstract}
${ }^{1} J$ ohanna Mäenpää, ${ }^{2}$ Bhavana Pothuri, ${ }^{3}$ Sileny Han, ${ }^{4}$ Dana Chase, ${ }^{5}$ David Bender, ${ }^{6}$ Philippe Follana, ${ }^{7}$ Emeline Bacque, ${ }^{7} \mathrm{LI}$ Yong, ${ }^{8}$ Antonio González-Martin, ${ }^{8}$ Bradley J Monk. ${ }^{1}$ Tampere University; ' Gynecologic Oncology Group (Gog); Perlmutter Cancer Center, Nyu Langone Health; Department of Obstetrics/Gynecology; ${ }^{3}$ University Hospitals Leuven; Department of Obstetrics and Gynecology; ${ }^{4}$ Arizona Oncology (US Oncology Network), University of Arizona College of Medicine; ${ }^{5}$ University of lowa; Department of Obstetrics and Gynecology; ${ }^{6}$ Gineco; Centre Antoine Lacassagne; ${ }^{7}$ GlaxosmithklineGrupo Español de Investigación En Cáncer de Ovario (Geico); Clínica Universidad de Navarra; Medical Oncology Department; ${ }^{8}$ Arizona Oncology (US Oncology Network), University of Arizona College of Medicine
\end{abstract}

\subsection{6/ijgc-2020-ESGO.114}

Background Niraparib is a poly(ADP-ribose) polymerase (PARP) inhibitor that is approved for use in heavily pretreated pts and as maintenance treatment of pts with newly diagnosed or recurrent ovarian cancer following a response to platinumbased chemotherapy (CT). Here we report PROs in pts receiving niraparib and placebo (PBO) in the PRIMA/ENGOTOV26/GOG-3012 trial.

Methods This double-blind, PBO-controlled, phase 3 study randomised 733 pts with newly diagnosed advanced ovarian, primary peritoneal, or fallopian tube cancer with a complete or partial response (CR or PR) to first-line (1L) platinum-based CT. Pts received niraparib or $\mathrm{PBO}$ once daily for 36 months or until disease progression. The primary endpoint was progression-free survival (PFS) assessed by blinded independent central review. PROs, a secondary endpoint, were collected every 8 weeks for 56 weeks, then every 12 weeks thereafter while treatment was ongoing. Once a pt discontinued treatment, PRO evaluations were performed at the time of treatment discontinuation and then at $4,8,12$, and 24 weeks ( \pm 1 week for each time point) after the end of treatment, regardless of the status of subsequent treatment. The validated PRO instruments utilised were FOSI, EQ-5D-5L, EORTC-QLQ-C30, and EORTC-QLQ-OV28.

Results Compliance rates were high for all of the PRO instruments used in the study. PRO analysis of the EORTC-QLQC30 and EORTC-QLQ-OV28 did not indicate a difference in health-related quality of life scores of pts treated with niraparib vs placebo. Mean scores between niraparib and placebo arms were similar at each time point. Overall, the health utility index showed a slight improvement trend in pts who received niraparib vs placebo.

Conclusion Consistent with PRO results in the NOVA study, pts receiving niraparib in the PRIMA trial did not experience a decrease in quality of life compared with those receiving placebo.

Disclosures Sponsor: GlaxoSmithKline, Waltham, MA, USA

NCT number: NCT02655016

Encore statement: This data is presented on behalf of the original authors with their permission. Presented at the European Society for Medical Oncology (ESMO) Annual Meeting, September 19-21, 2020, Virtual.

Dr. Mäenpää reports Honoraria from Tesaro, AstraZeneca, Clovis, Roche, MSD and OrionPharma

Dr. Pothuri reports grants, personal fees and non-financial support from GSK; Advisory Board fees from AstraZeneca and Clovis Oncology.

Dr. Chase reports speakers' bureau fees from GSK.

Dr. González-Martín reports personal fees and non-financial support from AstraZeneca; Grant and personal fees from GSK, Clovis Oncology, Roche Holding AG, Merck \& Co., Inc., Genmab, INMUNOGEN, Pharma Mar, S.A., and Oncoinvent AS.

Dr. Monk reports consulting and advisory role at Merck, GSK, Roche/Genentech, AstraZeneca, Advaxiz, Cerulean Pharma, Amgen, Immunogen, NuCana BioMed, Clovis Oncology, Pfizer, Mateon Therapeutics, Precision Oncology, Perthera, Abbvie, Myriad Pharmaceuticals, Incyte, VBL Therapeutics, Takeda, Samumed, Oncomed, OncoSec, ChemoID, Geistlich Pharma, Eisai and Chemocare; Speakers' bureau at Roche/Genentech, AstraZeneca, Janssen, Clovis Oncology and GSK; Honoraria from Merck, GSK, Roche/Genentech, AstraZeneca, Advaxis, Immunogen, NuCana BioMed, Clovis Oncology, Pfizer, Mateon Therapeutics, Precision Oncology, Pethera, Abbvie, Myriad Pharmaceuticals, Incyte, Janssen, Amgen, Genmab, Samumed, Takeda, VBL Therapeutics, Puma Biotechnology, Immunomedics, Conjupro Biotherapeutics, Agenus, OncoQuest, ChemoID, Geistlich Pharma, Eisai and Chemocare; and Research funding from Novartis, Amgen, 